\title{
Geração de Tecnologia em Universidades/Institutos de Pesquisa e a lmportância da Interação com Empresas: Constatações através da Base de Dados dos Grupos de Pesquisa do CNPq
}

\section{Technology Generation in University/Research Institutes and the Relevance of Interaction with Firms: Findings through the Directory of Research Groups (CNPq)}

\author{
Thiago Caliari* \\ Ulisses Pereira Santos** \\ Philipe Scherrer Mendes***
}

Resumo: O presente trabalho apresenta uma análise da relação entre a geração de tecnologia em universidades/institutos de pesquisa e a interação destas com empresas através da base de dados do diretório de grupos de pesquisa do CNPQ. Utilizando um modelo de dados em painel com informações dos cinco censos disponibilizados na base, é possível destacar, entre outros resultados, a relação positiva e altamente significante entre a geração de tecnologia e a interação entre os agentes, e as diferentes magnitudes e direção de influência para diferentes tipos de relacionamento, além da correlação positiva entre a geração de tecnologia e as publicações e escala dos grupos de pesquisa.

Palavras-chave: Interação universidade-empresa. Sistemas de inovação. Modelo de dados em painel. CNPq.

\begin{abstract}
The present work aims to study the relationship among technology created in universities and research institutes and their interactions with firms through available data from CNPq's Research Groups Directory. Using a panel data model with information from the five censuses available in base, we can highlight, among another results, a highly significant and positive relationship between technology generation and interaction between agents, and the different magnitudes and direction of influence for different types of relationships, besides the positive correlation between technology generation and publications and scale of the research groups.
\end{abstract}

Keywords: University-Industry interaction. Innovation systems. Panel data models. CNPq.

\footnotetext{
* $\quad$ Doutor em Economia pelo Centro de Desenvolvimento e Planejamento Regional (Cedeplar) da Universidade Federal de Minas Gerais (UFMG). Professor adjunto II do Instituto de Ciências Sociais Aplicadas (ICSA) da Universidade Federal de Alfenas (Unifal-MG). E-mail: thicaliari@ yahoo.com.br

** Doutor em Economia pelo Centro de Desenvolvimento e Planejamento Regional (Cedeplar) da Universidade Federal de Minas Gerais (UFMG). Professor adjunto A da Faculdade de Ciências Econômicas (FACE) da UFMG. E-mail: upesan@yahoo.com.br

*** Doutorando em Economia pelo Centro de Desenvolvimento e Planejamento Regional (Cedeplar) da Universidade Federal de Minas Gerais (UFMG).E-mail: philipescherrer@gmail.com
} 
JEL Classification: O3; C33.

\section{Introdução}

Suzigan e Albuquerque (2011) afirmam que, se forem analisados todos os casos de sucesso no desenvolvimento de produtos estritamente nacionais realizados no Brasil, que possuem vantagem comparativa internacional, pode-se encontrar um relacionamento entre as empresas que fabricam esses produtos e um instituto de pesquisa/universidade.

Mais do que essa afirmação a nível nacional, o estudo continuado de diferentes pesquisadores da área de Economia da Tecnologia tem apresentado a relevância do relacionamento entre esses agentes no desenvolvimento de novos produtos, novos processos, novas formas organizacionais, entre outros, em quaisquer níveis regionais delineados. Parece lógico, ainda ao se analisar o crescimento desse tipo de interação ao longo dos anos, que a busca de diferencial competitivo de um país, via inovação, passa com frequência sobre como esses agentes têm se relacionado na associação entre ciência e tecnologia.

No entanto, as interações entre universidades e empresas no Brasil são ainda limitadas ao se levar em conta o conjunto da economia brasileira. Os casos bem sucedidos de interação observados no país são exceção, dado o grau de imaturidade do sistema nacional de inovação. Como apontado pelas pesquisas sobre inovação sistematicamente realizadas no país, universidades e institutos de pesquisa ocupam posições apenas intermediárias entre as principais fontes de informação para a indústria nacional. Tal contexto gera uma desconexão entre os sistemas científico e tecnológico, além da subutilização das capacitações científicas nacionais, dada a qualidade e a produtividade das instituições de ensino e pesquisa.

Visto isso, este artigo pretende verificar a relevância dessas interações para o caso brasileiro através da análise da base de dados do Diretório dos Grupos de Pesquisa do Conselho Nacional de Desenvolvimento Científico e Tecnológico (CNPq). O intuito é entender (i) se a existência de interação entre universidades/institutos de pesquisa e empresas industriais provê melhores resultados sobre a geração de tecnologia dentro das universidades/institutos de pesquisa e (ii) quais os tipos de relacionamento que contribuem para esses resultados.

Além desta introdução, o trabalho apresenta outras seis seções. Na segunda seção, são discutidos aspectos teóricos da interação entre universidades/institutos de pesquisa e empresas; na terceira seção, é apresentado um breve histórico desse tipo de relacionamento no Brasil; na quarta seção, é apresentada a Base de Dados do Diretório dos Grupos de Pesquisa do CNPq; na quinta seção, discute-se sobre a técnica econométrica utilizada e os dois modelos sugeridos para análise; na sexta seção, são apresentados e discutidos seus resultados; e, por fim, na sétima seção, apresentam-se as considerações finais. 


\section{Interação Universidade/Institutos de Pesquisa e Empresas e a Geração de Tecnologia}

Não é recente a constatação de que o principal motor do desenvolvimento econômico é a inovação. As iniciativas teóricas com vistas à explicação do crescimento das nações sempre acabam rebatendo na dinâmica do progresso técnico, seja qual for a nomenclatura dada para tal (e são muitas, talvez por cada corpo teórico conflitante querer estabelecer seu próprio dicionário econômico). Entender, portanto, os determinantes do progresso técnico, suas nuances, as formas como as empresas - ou demais tipos de instituições - podem promulgá-la tem sido objeto de estudo de boa parte dos economistas - e demais cientistas sociais - para o entendimento de como determinada sociedade pode estar delineando o seu processo de desenvolvimento econômico.

Nesse anseio, tem crescido a relevância de análises que entendam o sistema institucional em variados níveis, como um sistema inter-relacional de apoio à promulgação de inovação, os chamados Sistemas de Inovação (SI). Os SIs podem ser entendidos como o conjunto de instituições, agentes e mecanismos de um país que contribuem para a criação, desenvolvimento e difusão das inovações tecnológicas, sendo seu conceito proposto inicialmente, e de maneira quase simultânea, pelos pesquisadores Bengt-Ake-Lundvall e Chris Freeman (ALBUQUERQUE, 2004).

O conceito de SI tem sido aplicado para diversos níveis de agregação, com importantes destaques para a análise a nível nacional (FREEMAN, 1988; FREEMAN, 1995; LUNDVALL, 1992; NELSON; ROSENBERG, 1993), a nível setorial (MALERBA, 2004) e até mesmo a nível regional (COOKE, 1998; LESTER, 2005; HUGHES, 2006; SANTOS, 2012).

O foco institucional é demasiado importante, mas de difícil definição. Conforme Edquist (2005), a "estrutura produtiva" e o "arranjo institucional" são destacados por Lundvall como as dimensões cruciais do sistema de inovação, enquanto outros pesquisadores - notadamente Nelson e Rosenberg - muitas vezes enfatizam as instituições e relações organizacionais que geram e difundem o conhecimento. Não há, portanto, uma definição exata dos limites institucionais de um SI.

Lundvall et al. (2009) apontam a relevância de toda a estrutura econômica específica do objeto de estudo para o delineamento das instituições relevantes. O ambiente de regulação nacional, as condições e estruturas do mercado, o sistema institucional e financeiro, dentre outros, tendem a modificar padrões, interações e instituições relevantes para o entendimento do SI. Afirmações assim apontam para a constatação de que teorizar fronteiras pode muitas vezes originar delimitações erradas. O mais sensato é a constatação empírica dos casos em estudo.

Essa dimensão de possibilidades de análise tem corroborado, contudo, para a afirmação da relevância de um tipo específico de interação entre agentes, notada- 
mente as relações entre agentes industriais e universidades/institutos de pesquisa. Apesar de ser o lócus comum da criação de inovação através de novos produtos, novos processos, melhoramentos, entre outros, a empresa industrial quase nunca realiza esse processo de maneira isolada; é comum a apresentação de experiências de inovações geradas com a ajuda de fornecedores, consumidores e, com ainda maior frequência, da relação com instituições que desenvolvem ciência.

Esse tipo de relacionamento não é novo. Nelson (1990) mostra que desde a segunda metade do século XIX as universidades possuem importância na geração de conhecimento para o crescimento econômico: na indústria química e de equipamento elétrico, as universidades forneciam pessoal técnico, e a pesquisa acadêmica era fonte de ideias para inovações em produto e processo. Nelson e Nelson (2002) apontam que o surgimento da indústria química sintética da Alemanha teve participação importante das interações entre universidades e firmas.

O fluxo ciência-tecnologia ${ }^{1}$ tem sido bastante explorado na literatura especializada. Talvez seja uma afirmação clara porque o processo inovativo, mais que qualquer outra atividade econômica, tem uma forte dependência do conhecimento científico. Como destaca Santos (2012), os fluxos de informações entre empresas industriais e universidades/institutos de pesquisa possibilitam a associação entre o conhecimento técnico-científico e o conhecimento produtivo, muitas vezes tácito, marcado por habilidades que se estabelecem por meio da rotina de produção dentro dos muros da própria fábrica, fazendo com que a inovação se efetive.

O conhecimento científico, por sua vez, é papel preponderante de universidades e institutos de pesquisa, seja na formação de recursos humanos especializados (ROSEMBERG e NELSON, 1994), seja na realização de pesquisas científicas - básicas ou aplicadas (NELSON, 1990; ROSEMBERG, 1992), ou até mesmo no nascimento de novas firmas spin-offs, especializadas em determinado campo científico (MALERBA, 2004).

Certo é que uma gama de trabalhos tem buscado explorar e entender essa relação. Mowery e Sampat (2005) apresentam em seu estudo a relevância das pesquisas acadêmicas para os avanços tecnológicos. Klevorick et al. (1995) e Cohen et al. (2002) utilizam pesquisas empíricas sobre a associação entre empresas e universidades através da Yale Survey e Carnegie Mellon Survey, respectivamente, apresentando resultados fundamentais para o entendimento e constatação da relevância desse relacionamento. Narin et al. (1997) analisam a citação de patentes americanas e, através disso, fornecem evidências empíricas da importância crescente da ciência pública universitária para as inovações tecnológicas das empresas industriais.

1 É importante destacar que, embora não seja o cerne do artigo, a causalidade contrária - tecnologia-ciência - também é importante, e tem relevante destaque nos trabalhos de Rosemberg (1982) e Moravcsik (1983), entre outros. 
Outros estudos analisam o fenômeno com focos diferenciados. Há as análises específicas de determinados setores industriais (NELSON, 1990; MANSFIELD, 1991), análises que buscam captar os impactos da proximidade regional sobre as interações (JAFFE et al., 1989; MANSFIELD e LEE, 1996; PAVITT, 1998; SANTOS, 2012), e ainda aqueles que analisam os efeitos diferenciados dessa interação em países em desenvolvimento (ALBUQUERQUE, 1999; SUTZ, 2000; RAPINI, 2007; RAPINI; CHIARINI; BITTENCOURT, 2012; SUZIGAN e ALBUQUERQUE, 2011). Esses últimos, aliás, apresentam a relevância da desconexão presente nas interações como um forte determinante da pequena competitividade apresentada pelas empresas industriais desses países.

Esses estudos têm certo intuito causal de um link específico entre ciência gerada nas universidades/institutos de pesquisa e tecnologia gerada dentro das firmas. Segundo Rapini et al (2012), ${ }^{2}$ o conhecimento científico é produzido nas universidades e institutos de pesquisa, sendo absorvido e acumulado como conhecimento tecnológico pelas firmas. Acontece, porém, que essa relação não é sempre uma relação unilateral, unívoca, com a interpretação básica de "geração de ciência na universidade e geração de tecnologia nas empresas".

Variados estudos têm apresentado a importância da geração e da transferência de tecnologia oriunda de universidades/institutos de pesquisa (vide, por exemplo, FRIEDMAN; SILBERMAN, 2003; SHANE, 2002; WRIGHT; BIRLEY; MOSEY, 2004; HARMON et al., 1997; MARKMANA et al., 2005). A tecnologia gerada nas universidades/institutos de pesquisa tem sido frequentemente observada como um importante gerador de novos negócios - spin-offs -, gerador do crescimento de empresas e até mesmo na criação de empregos. No âmbito de economias em desenvolvimento, tais instituições de ensino e pesquisa figurariam ainda como geradores de oportunidades de desenvolvimento e crescimento para a promoção e sustentação de processos de catch up (ALBUQUERQUE, 1999).

Frequentemente, esse fenômeno guarda um estreito link na relação das universidades/institutos de pesquisa com empresas industriais específicas. Harmon et al. (1997) apontam que a maior parte da tecnologia gerada na Universidade de Minessota entre 1983 e 1993 teve como destino o uso em grandes companhias, sendo objeto de melhoramento em produtos existentes ou a extensão de novas linhas de produtos. Shane (2002) mostra que as tecnologias geradas no Massachussets Institute of Technology (MIT), quando tornadas em patentes efetivas, geralmente são licenciadas para empresas não inventoras.

Lach e Schankerman (2008) apontam a relevância da capacidade infraestrutural da instituição em seu potencial inovativo: universidades americanas que

2 Visão esta que é considerada pelos próprios autores consistente com a estabelecida em Suzigan et al. (2011). 
possuem melhores condições de pesquisa tendem a fornecer melhores resultados tecnológicos.

Uma edição do conceituado Journal Management Science - volume 48, número 1, ano 2002 -, intitulada "University Entrepreneurship and Technology Transfer", foi inteiramente dedicada a estudos que analisaram a transferência de tecnologia das universidades e institutos de pesquisa para empresas. Dentre os estudos apresentados nessa edição, Colyvas et al. (2002) encontram um grande aumento no patenteamento e licenciamento de tecnologia feito por universidades após o Bayh-Dole Act de 1980 nos Estados Unidos, ${ }^{3}$ Mowery e Shane (2002), analisando também resultados após o Bayh-Dole Act, encontram que, nos últimos 20 anos, o número de universidades engajadas em licenciamento de tecnologia octuplicou, e o volume de patentes das universidades quadruplicou no mesmo período. Thursby e Thursby (2002) analisam a produtividade das universidades no licenciamento de novas tecnologias, e concluem que ela é positivamente correlacionada com a mudança comportamental dos pesquisadores da instituição - a disposição em permitir o licenciamento - e um aumento de relacionamento com demais instituições que realizam pesquisa e desenvolvimento (PED).

Friedman e Silberman (2003) apontam a relevância regional do relacionamento entre universidades/institutos de pesquisa e empresas de alta tecnologia como forma de manter vínculos estreitos e duradouros no desenvolvimento e transferência de novas tecnologias, resultado já também observado por Dorfman (1983) quando da análise da Route 128, em Boston, e do Vale do Silício, na Califórnia. Ainda, Harmon et al. (1997) encontram que $84 \%$ dos casos de transferência de tecnologia eram para empresas que possuíam relacionamento $a$ priori com a universidade. Lockett, Wright e Franklin (2003) encontram, baseados em um questionário para um grupo de 57 universidades inglesas, que as universidades com melhores resultados no que tange à criação e transferência de tecnologia são aquelas que possuem uma rede de relacionamento e experiência com demais instituições do SI, notadamente empresas industriais.

Ou seja, além de resultados que corroboram a relevância da geração de tecnologia nas universidades/institutos de pesquisa e posterior transferência para as empresas industriais, os vínculos obtidos por uma relação duradoura e estável entre esses agentes parecem ser fator determinante para uma relação profícua no que tange a criação de inovações.

3 O Bayh-Dole Act ou Emenda Constitucional de Patentes e Marcas (Pub. L. 96-517, 12/12/1980) é a legislação Americana em vigor que lida com a questão da propriedade intelectual. A principal mudança institucionalizada na emenda é a permissão dos direitos de propriedade de uma invenção financiada por recursos federais serem de posse dos inventores, como universidades, firmas e instituições sem fins lucrativos. 


\section{Breve Histórico da interação universidade-empresa no Brasil}

O Brasil apresenta limitações na interação entre universidades e empresas. Porém, a despeito disso, apresenta um histórico de interatividade bem sucedida em setores e regiões isoladas. Embora o caso com maior repercussão seja o da Embraer com o Centro de Tecnologia Aeronáutica (CTA), é possível verificar os primeiros indícios de interação universidade-empresa ainda no final do século XIX e no início do século XX.

A Escola de Minas de Ouro Preto, fundada em 1875, pode ser considerada a primeira instituição de ensino no país a se abrir para demandas do setor privado. Fundada na antiga capital do estado de Minas Gerais pelo metalurgista francês Henri Gorceix, a instituição tinha dentre suas propostas inovadoras para a época o objetivo de associar a formação teórica de seus alunos às práticas produtivas nos campos da mineração e da metalurgia. Nesse sentido, estudantes e professores tiveram participação direta no desenvolvimento da atividade siderúrgica no estado de Minas Gerais. Os principais canais de interação entre a Escola e o setor produtivo foram as análises mineralógicas e a execução de projetos industriais (CARVALHO, 2002). Dentre os resultados mais virtuosos dessa relação, é possível destacar a colaboração da Escola na elaboração do projeto do alto-forno da Companhia Siderúrgica Mineira, que começou a operar em 1920, sendo, então, o maior alto-forno já construído no país (SANTOS, 2012). Mais tarde, essa companhia foi incorporada pela Companhia Siderúrgica Belgo-Mineira, que foi a principal siderúrgica a atuar no Brasil até o surgimento da CSN, apenas na década de 1940. A Belgo-Mineira também contou com o apoio dos técnicos da Escola de Minas, principalmente no processo de adaptação ao uso do carvão vegetal.

Também no campo da mínero-metalurgia, é possível observar outro importante episódio de interação universidade-empresa no Brasil. Trata-se do relacionamento firmado entre Curso de Pós-graduação em Engenharia Metalúrgica de Materiais da UFMG e as empresas do setor. A partir de 1975, a instituição deu origem a cursos de pós-graduação stricto sensu em cooperação com empresas. Por meio dessa interação, técnicos alocados no setor produtivo tinham incentivos por parte das empresas a ingressarem nos cursos de mestrado e, posteriormente, doutorado, com vistas a contribuírem no avanço tecnológico delas através de suas descobertas na execução das pesquisas de final de curso (SANTOS, 2012). A contratação de pesquisas e o intercâmbio de profissionais também foram vias pelas quais ocorreu interação entre a universidade o setor produtivo.

No que tange a tecnologia agrícola, há um importante histórico de interações entre o setor produtivo e a Empresa Brasileira de Pesquisa Agropecuária, a Embrapa. A empresa foi criada em 1973 e logo estruturou uma rede de centros de pesquisa instalados ao longo do território nacional. Nesses centros foram desenvolvi- 
dos estudos nos campos das ciências do solo, melhoramento genético, recursos florestais, fisiologia, zoologia e nutrição animal, entre outros. $O$ ápice da interação da Embrapa com os produtores agropecuários brasileiros foi o desenvolvimento de uma tecnologia que adaptou o cultivo de grãos às condições climáticas do cerrado brasileiro, abrindo assim mais uma fronteira agrícola no país (SUZIGAN; ALBUQUERQUE, 2011). O sistema de inovação setorial da agropecuária no Brasil conta ainda com instituições como a Universidade Federal de Viçosa (UFV) e a Escola Superior de Agricultura Luiz de Queiros (ESALQ), entre outras, que contribuem para o desenvolvimento dessa atividade e mantêm uma história de atenção às demandas do setor produtivo.

Outros centros de pesquisa consolidados e interativos no país, mas atuando no campo da saúde, são o Instituto Butantan e o Instituto Oswaldo Cruz. Ambos contribuem historicamente para o desenvolvimento de vacinas e medicamentos para o controle de doenças tropicais. Sua intensa atividade científica e suas contribuições tecnológicas têm sido fundamentais para a posição de destaque alcançada pelo Brasil no campo das ciências da saúde (SUZIGAN; ALBUQUERQUE, 2011).

$\mathrm{Na}$ aeronáutica verifica-se, talvez, o mais emblemático caso de interação universidade-empresa no Brasil. Este é ligado à relação entre o sistema ITA-CTA e a Embraer. Em 1945, foram criados o Instituto de Tecnologia Aeronáutica e o Centro de Tecnologia Aeronáutica, sendo o primeiro destinado à formação de engenheiros aeronáuticos e o segundo voltado para a atividade de pesquisa. Eram, portanto, duas instituições com funções bem definidas, porém, interligadas. O sistema formado por essas instituições promoveu a chegada de pesquisadores estrangeiros ao Brasil e o aprendizado de tecnologias no setor, preparando o ambiente para a criação e consolidação da Empresa Brasileira de Aeronáutica, em 1969, um ano após o primeiro voo do protótipo do avião Bandeirante, desenvolvido pelo CTA (SUZIGAN; ALBUQUERQUE, 2011). Além de abrir caminho para a criação da Embraer, ao formar profissionais e promover o aprendizado no campo da ciência e tecnologia aeronáutica, essas instituições de ensino e pesquisa auxiliaram o desenvolvimento e fortificação da empresa que hoje figura entre as maiores produtoras de aeronaves do mundo.

Contudo, tais casos de interação bem sucedida são a exceção num sistema de inovação marcado pela baixa conexão entre as esferas científica e tecnológica. Ou seja, há ainda muita fragilidade no relacionamento entre universidades e empresas no Brasil, sendo os casos de sucesso marcados por uma trajetória histórica ou por fatores contextuais específicos. Nesse sentido, a literatura a respeito do tema, no Brasil, contempla uma série de limitadores das relações universidade-empresa, com destaque para a ausência de interlocutores adequados nas empresas, o seu baixo conteúdo inovador, a falta de instrumentos adequados para a comercialização de tecnologia e a pouca flexibilidade das instituições de ciência e tecnologia 
(RAPINI, 2007). Esse último aspecto decorre principalmente da rígida burocracia relativa aos processos de concessão e licenciamento de tecnologias desenvolvidas em universidades e institutos de pesquisa públicos para o setor privado. Tudo isso faz com que tais instituições sejam subaproveitadas como fonte de informação para a indústria brasileira. Essa condição se reflete no baixo percentual de empresas inovadoras que se relacionam com universidades no Brasil, o qual é bastante inferior àquele que se observa em países da Organização para a Cooperação e Desenvolvimento Econômico (OCDE) (RAPINI, 2007). Assim, prevalece no Brasil uma situação na qual, mesmo havendo razoável estrutura interna de ensino e pesquisa, atestada por uma representativa produção científica, não é devidamente aproveitada pela atividade industrial como insumo para a inovação, o que é uma característica de sistemas de inovação pouco desenvolvidos (ALBUQUERQUE, 1999).

Haveria, portanto, espaço para um melhor uso das interações universidade-empresa no Brasil com vistas à promoção de um maior desenvolvimento da indústria nacional. Partindo desse ponto de vista, são avaliadas na sequência as interações existentes no país e alguns de seus resultados, com base em dados do Diretório de Grupos de Pesquisa do CNPq.

\section{A Base de Dados do Diretório dos Grupos de Pesquisa do CNPq}

Este trabalho se valerá de dados relativos aos grupos de pesquisa cadastrados no CNPq contidos na base de dados do Diretório dos Grupos de Pesquisa (DGP) do referido conselho científico e tecnológico. A base constitui o inventário dos grupos de pesquisa em atividade no país, abrangendo informações sobre recursos humanos, linhas de pesquisa, especialidades do conhecimento, setores de atividade, produção científica e tecnológica dos pesquisadores, além dos padrões de interação com o setor produtivo. Esses grupos estão localizados em universidades, instituições isoladas de ensino superior, institutos de pesquisa científica, institutos tecnológicos, laboratórios de pesquisa e desenvolvimento de empresas estatais ou ex-estatais, e algumas organizações não governamentais com atuação em pesquisa.

Os grupos de pesquisa são organizados em hierarquia, com a presença de um líder pesquisador - ou dois, em alguns casos - e outros pesquisadores e estudantes que atuam em uma linha de pesquisa definida, coerente com a área de atuação do líder e que possua um vínculo de atividades à pesquisa com relativa permanência (CONSELHO NACIONAL DE DESENVOLVIMENTO CIENTÍFICO E

4 Assim como ocorre entre países desenvolvidos, os relacionamentos ocorrem predominantemente por meio de cooperações comerciais, como as que envolvem clientes, fornecedores ou outros entes da cadeia produtiva. No entanto, o destaque negativo para o caso brasileiro diz respeito à limitada interação com universidades, a qual se encontra muito abaixo do que ocorre em economias avançadas. 
TECNOLÓGICO, 2013a). A constituição de um grupo de pesquisa remete, dessa forma, à exigência de atuação contínua, o que evidencia a relevância da utilização desses dados como indicadores de conhecimento científico adquirido e utilizado nas universidades/institutos de pesquisa.

O DGP foi iniciado no CNPq em 1992 e, desde então, apresenta informações com frequência bienal da capacidade instalada de pesquisa no país, medida pelos grupos ativos em cada período. A partir de 2000, o site do DPG começa a apresentar informações censitárias e, excepcionalmente a partir de 2002, o DPG passou a conter informações dos padrões de interação desses grupos de pesquisa com o setor produtivo. ${ }^{5}$ Por esse motivo, os dados utilizados nesse trabalho são referentes aos censos de 2002 a 2010. Utilizaram-se para o trabalho consultas online no módulo 'Plano Tabular'.

Apresenta-se na Tabela 1 um levantamento descritivo inicial com o quantitativo de instituições, grupos de pesquisa, grupos de pesquisa que realizam interação e o total de relacionamentos, disponíveis na base para cada biênio censitário analisado.

Tabela 1 - Estatísticas descritivas básicas - Brasil

\begin{tabular}{l|c|c|c|c|c}
\hline $\begin{array}{l}\text { Agentes, grupos de pesquisa e } \\
\text { relacionamentos }\end{array}$ & $\mathbf{2 0 0 2}$ & $\mathbf{2 0 0 4}$ & $\mathbf{2 0 0 6}$ & $\mathbf{2 0 0 8}$ & $\mathbf{2 0 1 0}$ \\
\hline Instituições & 268 & 335 & 403 & 422 & 452 \\
\hline $\begin{array}{l}\text { Instituições com grupos de pesquisa } \\
\text { com relacionamento }\end{array}$ & 159 & 217 & 263 & 268 & 304 \\
\hline Grupos de pesquisa & 15158 & 19470 & 21024 & 22797 & 27523 \\
\hline $\begin{array}{l}\text { Grupos de pesquisa com relaciona- } \\
\text { mento }\end{array}$ & 1279 & 2151 & 2509 & 2726 & 3506 \\
\hline Empresas & 1791 & 2768 & 3352 & 3865 & 4995 \\
\hline Total de relacionamentos & 6129 & 9662 & 10346 & 10423 & 13113 \\
\hline
\end{tabular}

Fonte: Elaboração própria a partir do Diretório dos Grupos de Pesquisa do CNPq.

Verifica-se um aumento significativo na década pesquisada no número de instituições que possuem grupos de pesquisa (crescimento de 69\%), instituições com grupos de pesquisa que se relacionam (91\%), da quantidade de grupos de pesquisa (82\%), de grupos que realizam interação (174\%), do número de empresas que interagem (179\%) e da quantidade de interações realizadas (114\%).

Há de se destacar o significativo aumento dos grupos de pesquisa que se relacionam - e também do número de interações - em comparação ao aumento do

5 O conceito de empresa empregado pelo DGP/CNPq é bastante amplo, englobando não apenas empresas privadas com fins lucrativos, como também empresas estatais, associações, organizações não governamentais e agências públicas, entre outros. Desse modo, os dados do DGP/CNPq são utilizados aqui como proxy para os relacionamentos entre as instituições de pesquisa e o setor produtivo no Brasil. 
número de grupos de pesquisa, o que demonstra o maior engajamento científico em tarefas realizadas em conjunção com empresas. Ademais, essas últimas também apresentaram relevante incremento no engajamento de atividades de interação.

Assim, o trabalho valerá da informação dessas instituições durante os cinco biênios de análise para a proposição dos modelos de dados em painel que serão discutidos na próxima seção.

\section{A Técnica de Dados em Painel e os Modelos Sugeridos}

O exercício econométrico a ser realizado neste trabalho será com base no instrumental de painel de dados. Para tanto, suponhamos um modelo de regressão com $n$ observações, $K$ variáveis e $T$ períodos de tempo, expresso da seguinte forma:

$$
\begin{gathered}
y_{i t}=x_{i t} \beta+\varepsilon_{i t} \\
i=1,2, \ldots, n ; t=1,2, \ldots, T .
\end{gathered}
$$

Desse modelo teórico, tem-se: $y_{i t}$ como a variável dependente, $x_{i t}$ como um vetor $K x 1$ contendo as variáveis explicativas, $\beta$ como um vetor $K x 1$ de parâmetros a serem estimados e $\varepsilon_{i t}$ como os erros aleatórios $\left[\varepsilon_{i t} \sim i i d\left(0, \sigma^{2}\right)\right]$. Para a caracterização do painel de dados, usamos os subíndices $i$ e $t$ que denotam a unidade de observação e o período de tempo de cada variável, respectivamente, de forma que o número total de observações seja $n \times T$.

O componente de erro $\left(\varepsilon_{\mathrm{ii}}\right)$ tem a seguinte especificação:

$$
\varepsilon_{i t}=\mu_{\mathrm{i}}+\mathrm{v}_{\mathrm{it}}
$$

Em que $\mu_{\mathrm{i}}$ denota o efeito não observado, invariante no tempo, específico do indivíduo da análise, e $\mathrm{v}_{\mathrm{it}}$ denota o distúrbio remanescente, homocedástico e não autocorrelacionado $\left(\mu_{\mathrm{i}}\right.$ representa qualquer especificidade do indivíduo que não é incluída na regressão e que é invariante no tempo).

Entre as vantagens do uso da técnica de dados em painel estão (i) a captação e tratamento da heterogeneidade interna da unidade de observação e (ii) o aumento da efciência das estimativas pelo aumento do número de observações.

Dois modelos básicos podem ser derivados da utilização de dados em painel: o modelo de efeitos fixos (modelo FE) e o modelo de efeitos aleatórios (modelo RE).

No modelo de efeitos fixos, o erro não observado $\mu_{\mathrm{i}}$ é assumido fixo para cada indivíduo e o erro remanescente $\mathrm{v}_{\mathrm{it}}$ é considerado $\operatorname{IID}\left[\mathrm{v}_{\mathrm{it}} \sim \mathrm{iid}\left(0, \sigma^{2}\right)\right]$. O vetor de variáveis $x_{i t}$ é considerado independente de $v_{i t}$ para qualquer i e t. Modelos de efeitos fixos são utilizados na suposição de que os efeitos individuais $\mu_{\mathrm{i}}$ podem ser 
correlacionados com algum regressor de $x_{i t}$, o que, caso não se faça o devido tratamento metodológico, faz com que os estimadores fiquem enviesados.

Na proposição dos modelos de efeitos aleatórios (modelo RE), o erro não observado $\mu_{\mathrm{i}}$ é assumido como aleatório e IID $\left[\mu_{\mathrm{i}} \sim \operatorname{iid}\left(0, \sigma^{2}\right)\right]$, assim como o erro $v_{\mathrm{it}}$, sendo ainda $\operatorname{corr}\left(u_{i}, v_{i t}=0\right)$ e $\operatorname{corr}\left(x_{i t}, u_{i}, v_{i t}=0\right)$, ou seja, o termo de erro que representa uma característica não observável do indivíduo, invariante no tempo, não é correlacionado com nenhuma das outras variáveis do modelo e nem com o termo de erro $v_{i t}$.

Concluindo, o modelo de efeitos fixos permite a existência de correlação entre os efeitos individuais não observados com as variáveis explicativas. Entretanto, como destacam Loureiro e Costa (2009), se não se possui nenhuma suposição desse tipo, pode ser mais apropriado modelar esses efeitos como aleatoriamente distribuídos entre as unidades observacionais, utilizando o modelo de efeitos aleatórios.

A escolha entre qual dos dois tipos de modelo utilizar não é trivial. O debate sobre essa decisão é demasiado controverso, mas apresenta um grupo de testes e especificações que podem ser utilizados para ajudar nessa escolha. Para este trabalho, serão testados os dois modelos pelo teste de Hausman (1978), verificando ainda a possibilidade da especificação de Hausman e Taylor (1981) como alternativa de análise. ${ }^{6}$

Como o intuito do trabalho é verificar se a geração de tecnologia nas universidades/institutos de pesquisa é influenciada pelas interações entre essas instituições e empresas, faz-se a proposição da estimação de dois modelos econométricos:

1. Modelo de verificação pura das interações: nesse primeiro modelo será analisada a influência pura do número de grupos que interagem com empresas, controlado pelas devidas variáveis de controle;

2. Modelo de verificação dos tipos de relacionamento: nesse modelo analisar-se-ão como os diferentes tipos de relacionamento entre esses grupos e as empresas influenciam a geração de tecnologia.

Em ambos os modelos sugeridos, será utilizada a seguinte especificação econométrica:

$$
\operatorname{Tec}_{\mathrm{it}}=\beta_{\mathrm{i}} G P_{\mathrm{it}}+\theta_{\mathrm{i}} I U \mathrm{E}_{\mathrm{it}}+\kappa_{\mathrm{i}} \text { d.ano }
$$

Em que GP é um grupo de variáveis explicativas relacionadas aos grupos de pesquisa das instituições, IUE são informações relativas à interação universidade/

6 Como a especificação de Hausman e Taylor (1981) diz respeito à análise do modelo de efeitos fixos, essa alternativa só será testada se o teste de Hausman apresentar como melhor opção essa especificação de modelo. 
institutos de pesquisa e empresas e d.ano são variáveis dummy para cada censo apresentado. Essas variáveis explicativas, bem como a variável dependente, são apresentadas de maneira pormenorizada da seguinte forma:

a. Variável dependente Tec: corresponde à soma da produção tecnológica considerada especializada realizada nos grupos de pesquisa das instituições. Essa produção tecnológica considerada diz respeito às seguintes variáveis da base de dados: software com registro de patente; software sem registro de patente; produto tecnológico com registro de patente; produto tecnológico sem registro de patente; processo tecnológico com registro em catálogo; e processo tecnológico sem registro em catálogo.

O grupo intitulado GP contém as seguintes variáveis:

b. Grupos de pesquisa: número de grupos de pesquisa em atuação na instituição;

c. Fator_publicação: fator obtido através do método de Análise Fatorial,7 que engloba as seguintes publicações de pesquisadores ligados aos grupos de pesquisa das instituições:

Artigos nacionais: número de artigos publicados em periódicos nacionais;

Artigos internacionais: número de artigos publicados em periódicos internacionais;

Artigos em anais: número de artigos publicados em anais de congressos, simpósios, conferências, ou outros;

Liuros: número de livros publicados;

Capítulos de liuros: número de capítulos de livros.

d. Fator_recursos_humanos: fator obtido através do método de Análise Fatorial que engloba o quantitativo de recursos humanos (graduados, especialistas, mestres e doutores) ligados aos grupos de pesquisa das instituições;

Já o grupo intitulado IUE contém especificações com variáveis distintas para os dois tipos de modelo que se pretende apresentar, conforme apresentado no Modelo 1 e no Modelo 2:

7 Os valores da estimação dos fatores para publicações e recursos humanos encontram-se no anexo do artigo. 


\section{Modelo 1: Avaliação Pura das Interações}

a. Grupos interação: número de grupos de pesquisa na instituição que possuem interação com empresas;

b. Empresas interação: número de empresas que possuem interação com grupos de pesquisa da instituição.

\section{Modelo 2: Avaliação dos Tipos de Relacionamento}

a. Relacionamento 1...14: esse grupo contém a soma por tipo de relacionamento estabelecido entre os grupos de pesquisa da instituição e as empresas.

A especificação conceitual desses relacionamentos é apresentada na Tabela 2.

Tabela 2 - Tipos de relacionamento entre grupos de pesquisa e empresas

\begin{tabular}{l|l}
\hline $\begin{array}{c}\text { Nome do } \\
\text { Relacionamento }\end{array}$ & \multicolumn{1}{c}{ Descrição do Relacionamento } \\
\hline Relacionamento 1 & $\begin{array}{l}\text { Pesquisa científica sem considerações de uso imediato } \\
\text { dos resultados. }\end{array}$ \\
\hline Relacionamento 2 & $\begin{array}{l}\text { Pesquisa científica com considerações de uso imediato } \\
\text { dos resultados. }\end{array}$ \\
\hline Relacionamento 3 & $\begin{array}{l}\text { Atividades de engenharia não rotineiras, inclusive o } \\
\text { desenvolvimento de protótipo cabeça de série ou planta- } \\
\text {-piloto para o parceiro. }\end{array}$ \\
\hline Relacionamento 4 & $\begin{array}{l}\text { Atividades de engenharia não rotineiras, inclusive o } \\
\text { desenvolvimento/fabricação de equipamentos para o } \\
\text { grupo. }\end{array}$ \\
\hline Relacionamento 5 & $\begin{array}{l}\text { Desenvolvimento de software não rotineiro para o grupo } \\
\text { pelo parceiro. }\end{array}$ \\
\hline Relacionamento 6 & $\begin{array}{l}\text { Desenvolvimento de software para o parceiro pelo gru- } \\
\text { po. }\end{array}$ \\
\hline Relacionamento 7 & $\begin{array}{l}\text { Transferência de tecnologia desenvolvida pelo grupo } \\
\text { para o parceiro. }\end{array}$ \\
\hline Relacionamento 8 & $\begin{array}{l}\text { Transferência de tecnologia desenvolvida pelo parceiro } \\
\text { para o grupo }\end{array}$ \\
\hline Relacionamento 9 & $\begin{array}{l}\text { Atividades de consultoria técnica não contempladas nos } \\
\text { demais tipos. }\end{array}$ \\
\hline
\end{tabular}


conclusão.

\begin{tabular}{l|l}
$\begin{array}{c}\text { Nome do } \\
\text { Relacionamento }\end{array}$ & \multicolumn{1}{c}{ Descrição do Relacionamento } \\
\hline Relacionamento 10 & $\begin{array}{l}\text { Fornecimento pelo parceiro de insumos materiais para } \\
\text { as atividades de pesquisa do grupo sem vinculação a um } \\
\text { projeto específico de interesse mútuo. }\end{array}$ \\
\hline Relacionamento 11 & $\begin{array}{l}\text { Fornecimento pelo grupo de insumos materiais para as } \\
\text { atividades do parceiro sem vinculação a um projeto es- } \\
\text { pecífico de interesse mútuo. }\end{array}$ \\
\hline Relacionamento 12 & $\begin{array}{l}\text { Treinamento de pessoal do parceiro pelo grupo incluin- } \\
\text { do cursos e treinamento "em serviço". }\end{array}$ \\
\hline Relacionamento 13 & $\begin{array}{l}\text { Treinamento de pessoal do grupo pelo parceiro incluin- } \\
\text { do cursos e treinamento "em serviço". }\end{array}$ \\
\hline Relacionamento 14 & $\begin{array}{l}\text { Outros tipos predominantes de relacionamento que não } \\
\text { se enquadrem em nenhum dos anteriores. }\end{array}$ \\
\hline
\end{tabular}

Fonte: Elaboração própria a partir do Diretório dos Grupos de Pesquisa do CNPq.

O número de instituições utilizado nos dois modelos de dados em painel será diferente. Para o Modelo 1, serão consideradas apenas as instituições que possuíam grupos de pesquisa com relacionamento com empresas, em um total de 1211 observações, distribuídas nos cinco anos da análise. Já no Modelo 2, serão analisados todas as instituições dos cinco censos da análise (total de 1880 observações), pois pretende-se comparar a significância da existência dos 14 tipos de relacionamentos em relação a instituições que não possuíam nenhum desses tipos de relacionamento.

Ainda, como evidenciado na Tabela 1, o painel de dados utilizado pode ser classificado como um painel não balanceado, por não possuir o mesmo número de indivíduos em todos os períodos analisados. Segundo Wooldridge (2006), esse não pode ser considerado um problema; desde que a razão para a falta de dados de alguma observação i não esteja correlacionada com os erros idiossincráticos , esse painel não balanceado não causará problemas ao pesquisador.

\section{Resultados e Discussão}

Antes da apresentação dos resultados dos modelos, cabe destacar que a implementação do teste de Hausman define que a estimação dos parâmetros em um modelo de efeitos aleatórios é a melhor opção para os dois modelos sugeridos no 
artigo. ${ }^{8}$ Assim, apresentam-se, nos Quadros 1 e 2, os resultados e consequentes discussões para os dois modelos propostos, a iniciar pela apresentação do Modelo 1.

Quadro 1 - Modelo 1: avaliação pura das interações

\begin{tabular}{|c|c|c|c|}
\hline \multicolumn{4}{|c|}{$\begin{array}{c}\text { Variável dependente: Tec } \\
\text { Período: censos 2002, 2004, 2006, } 2008 \text { e } 2010 \\
\text { Número de observações: } 1211\end{array}$} \\
\hline Variável & Coeficiente & t-Statistic & Prob. \\
\hline Grupos de pesquisa & 0,414 & 10,47 & 0,000 \\
\hline Fator_publicação & 42,791 & 15,73 & 0,000 \\
\hline Fator_recursoshumanos & 3,158 & 6,34 & 0,000 \\
\hline Grupos Interação & 4,667 & 8,65 & 0,000 \\
\hline Empresas interação & 1,394 & 6,19 & 0,000 \\
\hline $\mathrm{d} 2004$ & 6,387 & 1,56 & 0,118 \\
\hline $\mathrm{d} 2006$ & 14,295 & 3,56 & 0,000 \\
\hline $\mathrm{d} 2008$ & $-3,391$ & $-0,83$ & 0,406 \\
\hline $\mathrm{d} 2010$ & 3,921 & 0,96 & 0,338 \\
\hline _cons & 0,528 & 4,11 & 0,000 \\
\hline $\mathrm{R}^{2}$ & 0,9384 & Wald $=$ & 70,05 \\
\hline $\mathrm{R}^{2}$ ajustado & 0,9239 & Prob $>\mathrm{cl}$ & $=0.00$ \\
\hline
\end{tabular}

Fonte: Elaboração própria.

Os resultados apresentados no Modelo 1 são interessantes por confirmarem empiricamente para o Brasil uma gama de proposições teóricas acerca da relação ciência-tecnologia. Inicialmente, pode-se notar que o aumento do número de grupos de pesquisa nas instituições (variável Grupos de Pesquisa) tem efeito positivo sobre a geração de tecnologia, mas o aumento do número de grupos que realizam interação com empresas (variável Grupos Interação) provoca resultados ainda mais satisfatórios para a instituição. Essa comparação de relevância pode ser feita pela comparação da magnitude do coeficiente, visto a significância estatística dessas duas variáveis.

A variável relativa ao quantum de empresas que possuem relação com grupos de pesquisa pertencentes à instituição - Empresas interação - também apresentou relevância positiva, ou seja, além de possuir interação, mostra-se importante no sentido institucional suscitar relacionamentos diversos, procurando estabelecer rede de relacionamento com grande número de empresas.

8 Os resultados do teste de Hausman para os dois modelos são apresentados nos anexos deste artigo. 
Outras informações relevantes podem ser extraídas da análise dos resultados do modelo. A primeira destas pode ser considerada a correlação positiva entre ciência e tecnologia institucional: a um maior número de publicações está associado um aumento da geração de tecnologia. Da mesma forma, o tamanho do grupo (escala de recursos humanos) influencia positivamente na tecnologia desenvolvida no âmbito da instituição.

No mais, as dummies de controle da evolução temporal da tecnologia desenvolvida nos grupos de pesquisa apresentam comportamentos distintos para os biênios analisados. A despeito da não significância estatística do coeficiente para o biênio 2004, 2008 e 2010, pode ser verificado que há um aumento apenas para o biênio 2006. A baixa significância estatística dessas dummies de controle corrobora o entendimento da relevância das variáveis sugeridas para a explicação da geração de tecnologia no modelo.

Pode-se pensar ainda em como os diferentes tipos de relacionamentos estabelecidos entre os grupos de pesquisa e as empresas podem contribuir para o incremento da tecnologia desenvolvida dentro do grupo. Para procurar responder essa indagação, apresenta-se o Modelo 2, que considera todas as variáveis de controle para as universidades (variáveis Grupo de Pesquisa, Fator_publicação e Fator_recursoshumanos) mais a inclusão dos 14 relacionamentos descritos na base de dados do diretório dos grupos de pesquisa do CNPq e apresentados anteriormente. Para esta análise, é importante ter em mente que seus resultados são relativos a todas as instituições, e não apenas àquelas que possuem grupos de pesquisa que interagem, conforme os resultados apresentados no Modelo 1. Os resultados são expressos no Quadro 2. 
Quadro 2 - Modelo 2: Avaliação dos Tipos de Relacionamentos

\begin{tabular}{|c|c|c|c|}
\hline \multicolumn{4}{|c|}{$\begin{array}{c}\text { Variável dependente: Tec } \\
\text { Período: censos 2002, 2004, 2006, } 2008 \text { e } 2010 \\
\text { Número de observações: } 1880\end{array}$} \\
\hline Variável & Coeficiente & t-Statistic & Prob. \\
\hline relacionamento 1 & 0,421 & 0,87 & 0,385 \\
\hline relacionamento 2 & 2,086 & 7,93 & 0,000 \\
\hline relacionamento 3 & 2,259 & 2,52 & 0,012 \\
\hline relacionamento 4 & 4,275 & 2,81 & 0,005 \\
\hline relacionamento 5 & 5,262 & 3,33 & 0,001 \\
\hline relacionamento 6 & $-2,802$ & $-2,66$ & 0,008 \\
\hline relacionamento 7 & 0,007 & 0,04 & 0,971 \\
\hline relacionamento 8 & 1,138 & 0,89 & 0,371 \\
\hline relacionamento 9 & $-1,563$ & $-3,16$ & 0,002 \\
\hline relacionamento 10 & 7,217 & 12,09 & 0,000 \\
\hline relacionamento 11 & $-2,155$ & $-2,37$ & 0,018 \\
\hline relacionamento 12 & 4,834 & 7,04 & 0,000 \\
\hline relacionamento 13 & 4,443 & 3,19 & 0,001 \\
\hline relacionamento 14 & 3,994 & 7,76 & 0,000 \\
\hline Grupos de pesquisa & 0,141 & 5,25 & 0,000 \\
\hline Fator_publicação & 36,28 & 15,37 & 0,000 \\
\hline Fator_recursoshumanos & 34,07 & 10,64 & 0,000 \\
\hline $\mathrm{d} 2004$ & 2,431 & 0,69 & 0,492 \\
\hline d2006 & 12,078 & 3,45 & 0,001 \\
\hline $\mathrm{d} 2008$ & $-1,916$ & $-2,28$ & 0,035 \\
\hline $\mathrm{d} 2010$ & $-0,001$ & $-2,38$ & 0,017 \\
\hline _cons & 17,715 & 5,06 & 0,000 \\
\hline $\mathrm{R}^{2}$ & 0,9181 & Wald $=$ & 95,66 \\
\hline $\mathrm{R}^{2}$ ajustado & 0,9066 & Prob $>$ & $=0.00$ \\
\hline
\end{tabular}

Fonte: Elaboração própria.

Os parâmetros para as variáveis de controle não apresentam modificações estruturais, e os resultados encontrados para os parâmetros dos diferentes tipos de relacionamento são dignos de nota. Como ponto inicial, pode-se constatar que os 
relacionamentos 2, 3, 4, 5, 10, 12, 13 e 14 apresentam significância estatística para o incremento tecnológico das instituições, de forma que instituições com grupos de pesquisa com esse tipo de relacionamento têm demonstrado desenvolver tecnologia em maior monta se comparado a instituições com grupos de pesquisa que não realizam interação.

Interessante ainda é a análise dos outros tipos de relacionamento, aqueles que não apontam relevância estatística para o incremento tecnológico ou aqueles que têm sentido contrário à intuição. Em relação ao primeiro caso, estamos falando dos relacionamentos do tipo 1, 7 e 8, pois tais não apresentam significância estatística, não sendo, portanto, relevantes para determinar o incremento inovativo dos grupos de pesquisa. Ou seja, grupos de pesquisa que realizam esse tipo de interação tendem a ter produtividade tecnológica da mesma monta de grupos de pesquisa que não realizam interação.

Há de se destacar os resultados para esses relacionamentos: o relacionamento 1 - pesquisa científica sem considerações de uso imediato dos resultados -, relacionamento 7 - transferência de tecnologia desenvolvida pelo grupo para o parceiro - e relacionamento 8 - transferência de tecnologia desenvolvida pelo parceiro para o grupo. Cabe lembrar que testes foram realizados com distintas especificações de variáveis de controle para o modelo, mas esse resultado em específico, como também os resultados encontrados para os demais relacionamentos, se manteve igual durante todos os testes.

Para o relacionamento 1, pode-se entender que a pesquisa científica sem consideração imediata não envolve focalização direta para resultados, não constitui o cerne da pesquisa para a inovação per se imediata, e por isso não corresponde exatamente a melhores resultados inovativos. Pode ainda, na maioria das vezes, estar ligada ao desenvolvimento de pesquisas básicas. Já o relacionamento 7 apresenta um resultado contra intuitivo, pois envolve a realização específica de transferências de tecnologia do grupo para o parceiro. Maiores estudos devem ser feitos para o entendimento desse resultado. O resultado do relacionamento 8 também é intuitivo, pois personifica um relacionamento específico para obtenção de tecnologia desenvolvida externamente e que, portanto, não tem relação direta com o aumento de tecnologia desenvolvida pelo grupo, pelo menos no curto prazo.

Os relacionamentos 6, 9 e 11 também são interessantes para análise, pois é apontado que os mesmos diminuem a geração tecnológica das instituições. Os relacionamentos 6, 9 e 11 dizem respeito, respectivamente, ao desenvolvimento de software para o parceiro pelo grupo, atividades de consultoria técnica e fornecimento de materiais e insumos pelo parceiro para o grupo.

A explicação para esses resultados parece ser de fácil entendimento e intuitiva. No primeiro caso - relacionamento 6-, o desenvolvimento específico de um software desenvolvido pela empresa para a instituição não vai personificar o 
desenvolvimento de uma nova tecnologia pelo grupo de pesquisa. Antes de tudo, foi realizada uma interação para que a instituição receba um novo produto desenvolvido por essa empresa, de forma que, de maneira clara, não se trata de um caso onde haja uma tecnologia desenvolvida internamente na instituição (assim como no caso do relacionamento 8). Já no relacionamento 9, a atividade de consultoria técnica não representa em si um relacionamento entre ciência e tecnologia; antes disso, apresenta uma relação de prestação de serviços especializados, pouco tendo a acrescentar, portanto, na geração de tecnologia dentro das instituições. ${ }^{9}$ Já o relacionamento 11 segue o mesmo raciocínio, visto que um relacionamento através do fornecimento de insumos do parceiro para o grupo personifica mais uma relação comercial do que uma interação para o desenvolvimento de tecnologia.

\section{Considerações Finais}

Em suma, o trabalho aqui realizado mostra que a interação entre universidades/institutos de pesquisa e empresas tem contribuído para a geração de novas tecnologias para as primeiras. Não obstante, no Modelo 1, a variável que indica o relacionamento entre esses agentes se apresenta com relevância para a geração de tecnologia. Segundo os resultados, o aumento dos grupos de pesquisa nas instituições incrementa a geração de novas tecnologias, mas quando esses grupos interagem com empresas, há um aumento ainda maior nessa geração. Esse resultado importante corrobora a relevância desse tipo específico de interação dentro do conceito dos sistemas de inovação. Outros resultados de cunho científico e tecnológico ainda são importantes de destacar, entre eles a correlação positiva entre a geração de tecnologia e (i) as publicações dos grupos de pesquisa e (ii) a escala dos grupos (representada pela quantidade de recursos humanos).

Além disso, no Modelo 2, as diferentes significâncias dos diferentes tipos de relacionamento atestam a relevância expressiva de determinadas relações em detrimentos de outras. Como já bastante discutido na literatura da área, alguns tipos de relacionamento, como a relação sem uso imediato de resultados, a consultoria técnica e fornecimento de materiais pouco - ou nada - tem a fornecer de respaldo para a geração de tecnologia. Em compensação, demais relacionamentos, como a realização de pesquisa para uso imediato e o treinamento de pessoal pelo grupo para o parceiro tendem a fornecer resultado positivo para o mesmo objetivo.

Cabe, porém, ressaltar as possíveis limitações das análises aqui apresentadas. Ao utilizar como unidade de análise a instituição e não diretamente os grupos de pesquisa, não se pôde verificar a influência das variáveis de controle para a

$9 \quad$ Arocena e Sutz (2003) apontam que universidades da América Latina possuem interações com firmas, em sua maior parte, compostas de consultoria técnica, e que esse tipo de relacionamento não personifica colaboração de pesquisa para criação tecnológica. 
geração de tecnologia sobre as diferentes áreas científicas de cadastro dos grupos; é de se esperar, por exemplo, que determinados tipos de relacionamento sejam mais importantes em determinadas áreas que em outras, e esse pode ser o futuro desenvolvimento do modelo utilizado. No momento, a limitação da base de dados não permitiu esse levantamento.

A despeito dessa limitação, acreditamos que esse tipo de análise quantitativa suscita resultados interessantes no que tange à análise das interações entre ciência e tecnologia no Brasil, tanto na verificação de resultados intuitivos quanto na análise daqueles que podem ser vistos, à primeira vista, como contra intuitivos. Não se pretende esgotar aqui as discussões e possibilidades de estudo dessas relações tão importantes para o desenvolvimento tecnológico de uma nação; antes disso, o intuito é de despertar o conhecimento e verificação.

\section{Referências}

ALBUQUERQUE, E. M. National systems of innovation and non-OCED countries: notes about a rudimentary and tentative "tipology". Brazilian Journal of Political Economy, v. 19, n. 4 (76), p. 35-52, Oct./Nov. 1999.

The national system of innovation in historical perspective. Revista Brasileira de Inovação, Rio de Janeiro, v. 3, n. 1, p. 9-13, 2004.

AROCENA, R.; SUTZ, J. Knowledge, innovation and learning: systems and policies in the north and in the south. In: CASSIOLATO, J. E.; LASTRES, H. M. M.; MACIEL, M. L. (Ed.). Systems of Innovation and Development: Evidence from Brazil. Northampton, MA, USA: Edward Elgar, 2003.

BALTAGI, B. H. Econometric analysis of panel data. West Sussex, England: John Wiley, 2005.

CARVALHO, J. M. A escola de minas de ouro preto: o peso da glória. 2. ed. Belo Horizonte: Editora da UFMG, 2002.

COHEN, W. M.; NELSON, R. R.; WALSH, J. P. The influence of public research on industrial RED. Management Science, v. 48, n. 1, p. 1-23, Jan. 2002.

COLYVAS, J. et al. How do university inventions get into practice? Management Science, v. 48, n. 1, p. 61-72, Jan. 2002.

CONSELHO NACIONAL DE DESENVOLVIMENTO CIENTÍFICO E TECNOLÓGICO. Informações gerais sobre o diretório dos grupos de pesquisa do CNPq. 2013. Disponível em: $<$ http://dgp.cnpq.br/censos/inf_gerais/index_que_eh.htm >. Acesso em: 10 maio 2013.

COOKE, P. Introduction: origins of the concept. In: BRACZYK, H.; COOKE, P.; HIDERNREICH, M. (Ed.). Regional Innovation Systems. London: UCL Press, 1998. p. 2- 25.

DORFMAN, N. S. Route 128: the development of a regional high technology economy. Research Policy, v. 12, n. 6, p. 299-316, 1983. 
EDQUIST, C. Systems of innovation: perspectives and challenges. In: FARGERBERG, J.; MOWERY, D.; NELSON, R. (Ed.). The Oxford handbook of innovation. Oxford: Oxford University, 2005. p. 181-208.

FREEMAN, C. Japan: a new national system of innovation? In: DOSI, G. et al. (Ed.). Technical change an economic theory. London: Printer, 1988. p. 330-348.

. The "national system of innovation" in historical perspective. Cambridge Journal of Economics, v. 19, n. 1, p. 5-24, 1995.

FRIEDMAN, J., SILBERMAN, J. University technology transfer: do incentives, management, and location matter? The Journal of Technology Transfer, v. 28, n. 1, p. 17-30. Jan. 2003.

HARMON, B. et al. Mapping the university technology transfer process. Journal of Business Venturing, v. 12, n. 6, p. 423-434, Nov. 1997.

HAUSMAN, J. A. Specification tests in econometrics. Econometrica, v. 46, n. 6, p. 1251-1271, 1978.

HAUSMAN, J. A.; TAYLOR, W. E. Panel data and unobservable individual effects. Econometrica, v. 49, n. 6, p. 1377-1398, 1981.

HUGHES, A. University-industry linkages and UK science and innovation policy. Cambridge, Massachusetts: University of Cambridge, June 2006. (Working Paper, n. 326).

JAFFE, A. B. Real effects of academic research. The American Economic Review, v. 79, n. 5, p. 957-970, Dec. 1989.

KLEVORICK, A. K. et al. On the sources and significance of inter-industry differences in technological opportunities. Research Policy, v. 24, n. 2, p. 185-205, Mar. 1995.

LACH, S.; SCHANKERMAN, M. Incentives and inventions in universities. Cambridge, Massachusetts: National Bureau of Economic Research, May 2003. (Working Paper, n. 9727).

LESTER, R. K. LIS Project - Phase I findings: overview and discussion. Massachusetts: MIT IPC Local Innovation Systems, Dec. 2005. (Working Paper, n. 05-004).

LOCKETT, A.; WRIGHT, M.; FRANKLIN, S. Technology transfer and University spin-out estrategies. Small Business Economics, v. 20, n. 2, p. 185-200, 2003.

LOUREIRO, A. O. F.; COSTA, L. O. Uma breve discussão sobre os modelos com dados em painel. IPECE: Ceará, 2009. (Nota Técnica 37).

LUNDVALL, B. National systems of innovation: towards a theory of innovation and interactive learning. London: Pinter, 1992. 342 p.

LUNDVALL, B. A. et al. Innovation system research and developing countries. In: LUNDVALL, B. A. et al. Handbook of innovation systems and developing countries: building domestic capabilities in a global setting. Cheltenham, UK: Edward Elgar, 2009.

MALERBA, F. Sectoral systems of innovation: concepts, issues and analyses of six major sectors in europe. Cambridge: Cambridge University Press, 2004. 
MANSFIELD, E. Academic research and industrial innovation. Research Policy, v. 20, n. 1, p. 1-12, Feb. 1991.

MANSFIELD, E.; LEE, J. The modern university: contributor to industrial innovation and recipient of industrial PEBD support. Research Policy, v. 25, n. 7, p. 1047-1058, Oct. 1996.

MARKMANA, G. D. et al. Innovation speed: transferring university technology to market. Research Policy, v. 34, n. 7, p. 1058-1075. Sept. 2005.

MORAVCSIK, M. The role of science in technology transfer. Research Policy, v. 12, n. 5, p. 287-296, Oct. 1983.

MOWERY, D. C.; SHANE, S. Introduction to the special issue on university entrepreneurship and technology transfer. Management Science, v. 48, n. 1, p. 5-9, Jan. 2002.

MOWERY, D.; SAMPAT, B. Universities in national innovation systems. In: FARGERBERG, J; MOWERY, D.; NELSON, R. (Ed.). The Oxford handbook of innovation. Oxford: Oxford University, 2005. p. 209-239.

NARIN, F.; HAMILTON, K. S.; OLIVASTRO, D. The increasing linkage between U.S. technology and public science. Research Policy, v. 26, n. 3, p. 317-330, 1997.

NELSON, R. Capitalism as an engine of progress. Research Policy, v. 19, n. 3, p. 193-214, June 1990.

NELSON, R. R.; ROSENBERG, N. Techical innovation and national systems. In: NELSON, R. (Ed.). National innovation systems: a comparative analysis. New York, Oxford: Oxford University, 1993. p. 76-114,

NELSON, R. R.; NELSON, K. Technology, institutions, and innovation systems. Research Policy, v. 31, n. 2, p. 265-272, 2002.

PAVITT, K. The social shaping of the national science base. Research Policy, v. 27, n. 8, p.793805, 1998.

PÓVOA, L. M. C. Patentes de universidades e institutos públicos de pesquisa e a transferência de tecnologia para empresas no Brasil. 2008. 153 f. Tese (Doutorado em Economia) - Centro de Desenvolvimento e Planejamento Regional, Universidade Federal de Minas Gerais, Belo Horizonte, 2008.

RAPINI, M. S. Interação universidade-empresa no brasil: evidências do diretório dos grupos de pesquisa do CNPq. Estudos Econômicos, São Paulo, v. 37, n. 1, p. 211-233, jan./mar. 2007.

RAPINI, M. S.; CHIARINI, T.; BITTENCOURT, P. F. The University-Industry relations in a peripheral context: their contribution to knowledge generation and innovation in Brazil. In: CONGRESO ANUAL DE LA ASOCIACIÓN DE ECONOMÍA PARA EL DESARROLLO DE LA ARGENTINA, 4., 2012, Buenos Aires. Anales... Buenos Aires: AADA, 2012.

ROSENBERG, N. Inside the black box: technology and economics. Cambridge: Cambridge University, 1982. 
ROSENBERG, N. Scientific instrumentation and univeristy research? Research Policy, v. 21, n. 4, p. 381-390, Aug. 1992.

ROSENBERG, N.; NELSON, R. American university and technical advance in industry. Research Policy, v. 23, n. 3, p. 323-348, May 1994.

SANTOS, U. P. Ambiente institucional e inovação na siderurgia de Minas Gerais. Fortaleza: Editora do Banco do Nordeste, 2012.

SHANE, S. Selling university technology: patterns from MIT. Management Science, v. 48, n. 1, p. 122-137, 2002.

SUTZ, J. The new role of the university in the productive sector. In: ETZKOWITZ, H.; LEYDESDORFF, L. Universities and the global knowledge economy: a triple helix of universityindustry-government. London: Cassell Academic,, 2000.

SUZIGAN, W.; ALBUQUERQUE, E. The underestimated role of universities for the Brazilian system of innovation. Revista de Economia Política, v. 31, n. 1, p. 3-30, 2011.

SUZIGAN, W.; RAPINI, M. S.; ALBUQUERQUE, E. M. A changing role for universities in the periphery. Minas Gerais: Cedeplar/UFMG, abr. 2011. (Texto para Discussão, n. 420).

THURSBY, J. G.; THURSBY, M. C. Who is selling the ivory tower? Sources of growth in university licensing. Management Science, v. 48, n. 1, p. 90-104, Jan. 2002.

WOOLDRIDGE, J. M. Introductory econometrics: a modern approach. 3. ed. Mason: Thomsom, 2006.

WRIGHT, M.; BIRLEY, S.; MOSEY, S. Entrepreneurship and university technology transfer. The Journal of Technology Transfer. v. 29, n. 3-4, p 235-246, Aug. 2004. 
Anexo A - Análise fatorial - Fator_Publicação

\begin{tabular}{|c|c|c|c|c|}
\hline Ordem & Autovalor & \multicolumn{2}{|c|}{ Porcent. explicada } & $\begin{array}{c}\text { Porcent. } \\
\text { Acumulada }\end{array}$ \\
\hline Fator 1 & 4,58533 & \multicolumn{2}{|c|}{$100,17 \%$} & $100,17 \%$ \\
\hline Fator 2 & 0,02986 & \multicolumn{2}{|c|}{$0,65 \%$} & $100,82 \%$ \\
\hline Fator 3 & 0,01670 & \multicolumn{2}{|c|}{$0,36 \%$} & $101,18 \%$ \\
\hline Fator 4 & $-0,02112$ & \multicolumn{2}{|c|}{$-0,46 \%$} & $100,72 \%$ \\
\hline Fator 5 & $-0,03304$ & \multicolumn{2}{|c|}{$-0,72 \%$} & $100,00 \%$ \\
\hline Variável & Fator 1 & Fator 2 & Fator 3 & Uniqueness \\
\hline Artigos Nacionais & 0,9746 & 0,0616 & $-0,0510$ & 0,0438 \\
\hline Artigo Internacional & 0,9584 & $-0,1055$ & $-0,0492$ & 0,0680 \\
\hline $\begin{array}{l}\text { Artigos em anal de } \\
\text { congresso }\end{array}$ & 0,9645 & 0,0924 & $-0,0263$ & 0,0606 \\
\hline Livro & 0,9068 & 0,0281 & 0,0997 & 0,1669 \\
\hline Capítulo de Livro & 0,9821 & $-0,0749$ & 0,0323 & 0,0288 \\
\hline \multicolumn{5}{|c|}{ Número de observações: 1880} \\
\hline \multicolumn{5}{|c|}{ Teste LR: $\operatorname{chi} 2(10)=1,7 \mathrm{e} 04$ Prob $>$ chi $2=0.0000$} \\
\hline
\end{tabular}

Fonte: Elaboração própria. 
Anexo B - Análise fatorial - Fator_Recursos humanos

\begin{tabular}{|c|c|c|c|c|}
\hline Ordem & \multicolumn{2}{|c|}{ Autovalor } & $\begin{array}{l}\text { Porcent. } \\
\text { explicada }\end{array}$ & $\begin{array}{c}\text { Porcent. } \\
\text { Acumulada }\end{array}$ \\
\hline Fator 1 & \multicolumn{2}{|c|}{2,75186} & $102,77 \%$ & $102,77 \%$ \\
\hline Fator 2 & \multicolumn{2}{|c|}{0,08038} & $3,00 \%$ & $105,77 \%$ \\
\hline Fator 3 & \multicolumn{2}{|c|}{0,01095} & $0,41 \%$ & $106,18 \%$ \\
\hline Fator 4 & \multicolumn{2}{|c|}{$-0,16555$} & $-6,18 \%$ & $100,00 \%$ \\
\hline Variável & Fator 1 & Fator 2 & Fator 3 & Uniqueness \\
\hline Graduado & 0,8520 & $-0,0405$ & 0,0733 & 0,2671 \\
\hline Especialista & 0,8754 & $-0,1805$ & $-0,0156$ & 0,2008 \\
\hline Mestre & 0,8537 & 0,0465 & $-0,0712$ & 0,2639 \\
\hline Doutor & 0,7285 & 0,2098 & 0,0165 & 0,4250 \\
\hline
\end{tabular}

Número de observações: 1880

Teste LR: $\operatorname{chi} 2(6)=5151,80$ Prob $>$ chi2 $=0.0000$

Fonte: Elaboração própria. 
Anexo C - Teste de Hausman - Modelo 1

\begin{tabular}{|c|c|c|c|c|}
\hline Variável & $\begin{array}{c}\text { (b) } \\
\text { Efeito Fixo }\end{array}$ & $\begin{array}{c}\text { (B) } \\
\text { Efeito } \\
\text { Aleatório }\end{array}$ & $\begin{array}{c}\text { (b-B) } \\
\text { Diferença }\end{array}$ & $\begin{array}{c}\text { sqrt(diag(V_b-V_B }) \text { ) } \\
\text { S.E. }\end{array}$ \\
\hline $\begin{array}{l}\text { Grupos de } \\
\text { pesquisa }\end{array}$ & 0,651 & 0,414 & 0,237 & 0,084 \\
\hline $\begin{array}{l}\text { Fator_publi- } \\
\text { cação }\end{array}$ & 38,384 & 42,791 & $-4,406$ & 1,182 \\
\hline $\begin{array}{l}\text { Fator_recur- } \\
\text { sos humanos }\end{array}$ & $-4,284$ & 3,158 & $-7,441$ & 4,822 \\
\hline $\begin{array}{l}\text { Interação } \\
\text { grupo }\end{array}$ & 5,149 & 4,667 & 0,482 & 0,383 \\
\hline $\begin{array}{l}\text { Empresas } \\
\text { interação }\end{array}$ & 1,448 & 1,394 & 0,054 & 0,171 \\
\hline d2004 & 2,035 & 6,387 & $-4,352$ & - \\
\hline $\mathrm{d} 2006$ & 8,579 & 14,295 & $-5,717$ & 1,037 \\
\hline $\mathrm{d} 2008$ & $-11,479$ & $-3,391$ & $-8,088$ & 1,384 \\
\hline $\mathrm{d} 2010$ & $-6,975$ & 3,921 & $-10,896$ & 2,108 \\
\hline \multicolumn{5}{|c|}{$\mathrm{b}=$ consistente sob Ho e Ha. } \\
\hline \multicolumn{5}{|c|}{$\begin{array}{c}\text { B = inconsistente sob Ha, eficiente sob Ho. } \\
\text { Teste: Ho: diferenças nos coeficientes não são sistemáticas } \\
\text { chi2 }(17)=45,98\end{array}$} \\
\hline
\end{tabular}

Fonte: Elaboração própria. 
Anexo D - Teste de Hausman - Modelo 2

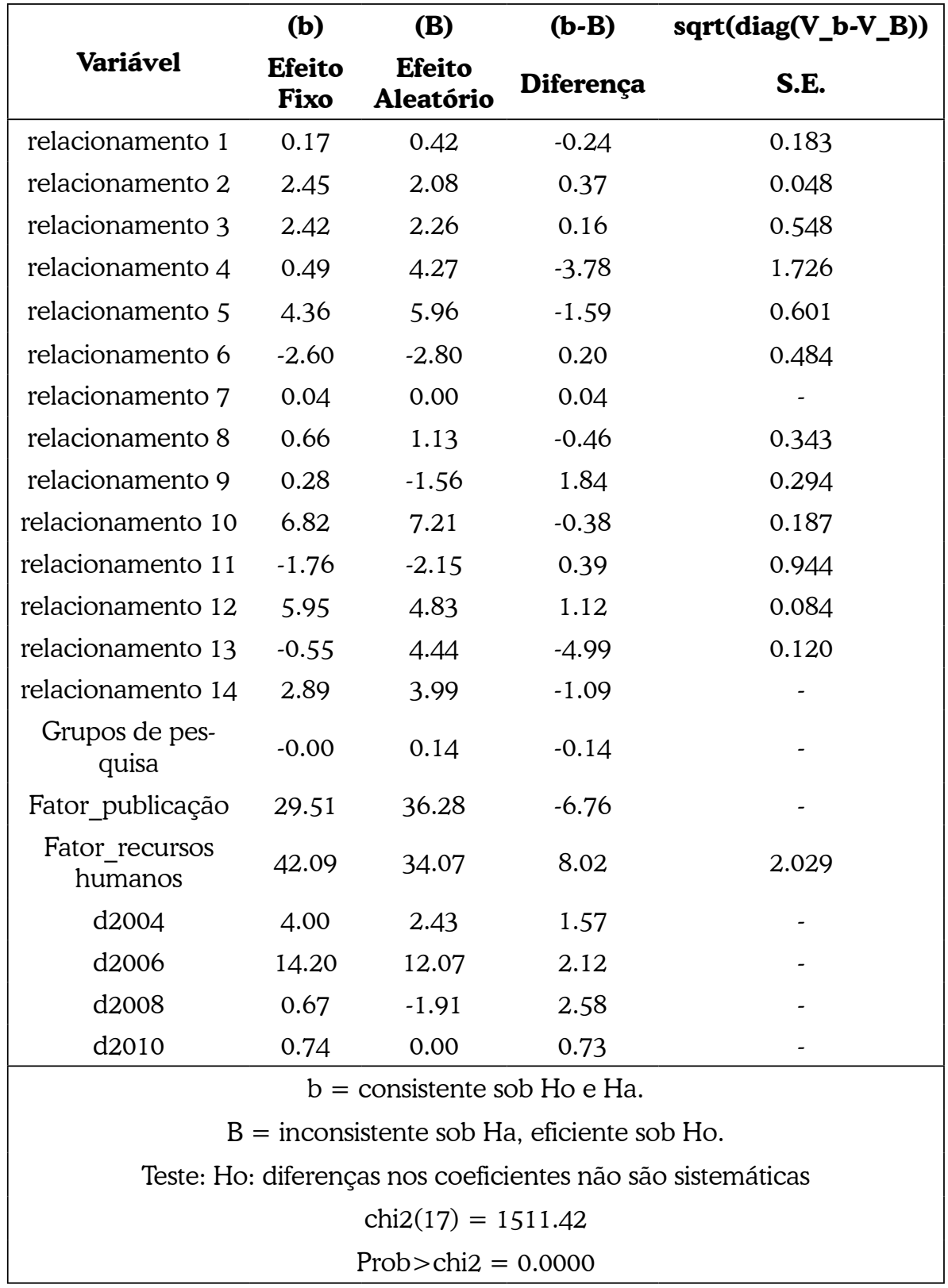

Fonte: Elaboração própria.

Recebido em: 12/03/2015. Aceito em: 22/09/2015. 